\title{
FERMILAB-CONF-05-118-AD-CD A BETATRON TUNE FITTING PACKAGE FOR THE TEVATRON 21.4 MHZ SCHOTTKY*
}

\author{
Paul L. G. Lebrun ${ }^{\dagger}$, Tanaji Sen, Jianming You, Zongwei Yuan, Fermilab, Batavia, IL 60510, U. S.A. \\ Ezio Todesco, CERN, Geneva, Switzerland
}

\section{Abstract}

The Fermilab Tevatron is equipped with two independent Schottky monitors for measurement of betatron tunes, one operating at $21.4 \mathrm{MHz}$ and the other at $1.7 \mathrm{GHz}$. A new front-end and related data acquisition for the $21.4 \mathrm{MHz}$ resonator has been installed and commissioned during the FY04 Collider RunII. Sophisticated fitting strategies are required to analyze the spectra. Optimization of this fitting package allows us to report tune and chromaticity measurements at almost $1 \mathrm{~Hz}$.

\section{INTRODUCTION}

The Fermilab Tevatron Collider performance depends critically on the accurate measurements of betatron tunes and chromaticities [1]. The Tevatron is now equipped with three distinct devices that are able to report betatron tunes: the old 21.4 MHz resonator [3], the new 1.7 GHz Schottky monitor [2] and BPMs or strip-lines connected to new electronics [4]. Such systems are complementary to each other. Their use differs for the two types of circulating beams labeled "coalesced" or "uncoalesced". Uncoalesced beam consists of a train of $\approx 20$ successive $53 \mathrm{MHz}$ bunches with comparatively small longitudinal emittance. They are used to tune the Tevatron during preparations for a luminosity store. Such trains can be coalesced into single bunches in the Main Injector. These coalesced bunches, which are used for luminosity stores, have higher intensities and larger longitudinal emittances than uncoalesced bunches. Given the machine and Schottky monitor parameters listed in Table 1, the betatron signal line shape will be much broader for the $1.7 \mathrm{GHz}$ system, preventing observation of individual synchrotron lines, even with uncoalesced beams. Conversely, the $21.4 \mathrm{MHz}$ cavity has a higher Q then the $1.7 \mathrm{GHz}$ cavity and therefore will integrate particle or coherent beam motion for a significant fraction of a Tevatron turn, preventing the tunes of consecutive coalesced bunches from being observed individually. The 1.7 GHz monitor has this capability [2].

Despite a successful installation of the $1.7 \mathrm{GHz}$ system, the $21.4 \mathrm{MHz}$ system was found to be very useful for making precise measurements of tune and chromaticities with uncoalesced beams. This paper presents a recent upgrade of the Tevatron $21.4 \mathrm{MHz}$ Schottky and the analysis of tune spectra. An early use of this method for measuring the

\footnotetext{
${ }^{*}$ Work supported by DOE under contract DE-AC02-76CH03000 with the U.S. Dept. of Energy.

†lebrun@fnal.gov
}

chromaticity was reported in Run I of the Tevatron [3].

\section{THE ICS-110B/VME FRONT-END}

In 2003, the old HP3561A spectrum analyzer was read via a GPIB interface. This system was slow, with a lag-time of $\approx 5$ seconds, and sometimes unreliable. In 2004, this antiquated front-end was replaced with a VME crate located in the A1 service building. Signals from the frequency down-converter (from $21.4 \mathrm{MHz}$ to the betatron base-band of $\approx 27 \mathrm{kHz}$ ) are multiplexed such that other control systems could function in parallel with our new tune-fitter front-end. This new system consists of a ICS-110B digitizer board[5] and a Motorola MVME-2434 Data Acquisition board running VxWork [6]. The four down-converted signals (proton/antiproton, horizontal/vertical pick-ups) are digitized concurrently at $100 \mathrm{kHz}$. This frequency is more than twice the betatron base-band, avoiding aliasing confusion. Approximately 4 sweeps of 20,000 samples each are stored every second for each of the 4 channels. Fast Fourier transforms are performed on the MVME-2400 board, using the FFTW package[7]. Relevant portions of the frequency spectrum, centered on the average betatron lines (corresponding to a fractional betatron tune of 0.575 ), are send via ACNET to two computing nodes running Linux, where the real-time tune spectra fitting is performed. In summary, the analog to digital conversion as well as the $\mathrm{i} / \mathrm{o}$ intensive part of this system is done on VME, while the CPU demanding part of the analysis is done on cheaper computing nodes, running Open Source software, such as the ROOT fitting package[8].

\section{REAL-TIME ANALYSIS AND CHROMATICITY FORMALISM}

The tune fitter package consists of a collection of fitting functions and algorithms to smear, smooth or average arbitrary number of spectra[9]. The data for uncoalesced beams is fitted with a incoherent sum of five Lorentzian (aka "Breit-Wigner") functions:

$$
A(f)=B+\sum_{k=-2}^{2}\left(A_{k} \Gamma /\left(\left(f-f_{0, k}\right)^{2}+\Gamma^{2} / 4\right)\right.
$$

where $f_{0}$ is the frequency of the main line, $f_{0,(k+1)}-f_{0, k}$ is the synchrotron tune split, $\Gamma$ is the width of the spectral line. The strength of each individual line $A_{k}$ and $B$ the noise floor are free parameters. If the noise is relatively small, the frequency at the central line $k=0$ is equal to 
the tune. Under the assumptions specified below, the ratios $A_{k} / A_{0}, k \neq 0$ depend on the chromaticity as:

$$
A_{k} \propto e^{-z_{k} / 2} \sqrt{I_{k}\left(z_{k}\right)}
$$

where $I_{k}$ are the modified Bessel functions and $z_{k}$ is defined as $z_{k}=\left(2 \pi \sigma_{t}\left(m \omega_{r}+k \omega_{s}-\omega_{\beta} \xi / \eta\right)\right)^{2}$ Here, $\sigma_{t}$ is the rms bunch length, $m$ is the revolution harmonic corresponding to the resonant frequency of the Schottky monitor, $\omega_{r}$ the angular revolution frequency, $\omega_{s}$ the synchrotron frequency, $\omega_{\beta}=Q_{\beta} \omega_{r}$, the betatron frequency. $\xi=Q_{\beta}^{\prime} / Q_{\beta}$ is the scaled chromaticity and $\eta$ is the slip factor. Table 1 list the parameter values.

The assumptions in deriving the expressions above are: i) Time averages can be replaced by phase space averages (ergodic hypothesis). ii) Distributions in phase space are Gaussian, iii) The transverse tune $Q_{\beta}$ depends linearly on the particle momenta via the linear chromaticity, iv) $Q_{\beta}$ is independent of the transverse amplitude, v) The longitudinal emittance is small enough such that the synchrotron motion is a linear oscillation at a fixed frequency vi) The higher order terms stemming from the synchrotron motion are neglected. Most of these assumptions are not essential and can be relaxed.

Table 1: Parameters for the 21.4 MHz Schottky Monitor, uncoalesced beams

\begin{tabular}{|l|c|c|}
\hline Parameter & Value (150 GeV) & Value (980 GeV) \\
\hline Harmonic, $\mathrm{m}$ & 448 & 448 \\
$f_{r}(\mathrm{kHz})$ & 47.711 & 47.713 \\
Loaded $Q_{l}$ & 370 & 370 \\
$\sigma_{t}(\mathrm{~ns})$ & 0.6 & 0.3 \\
$f_{s}(\mathrm{~Hz})$ & 85 & 31 \\
$Q_{\text {beta }}$ & 20.57 to 20.59 & 20.57 to 20.59 \\
$Q^{\prime},(\mathrm{X}$ or $\mathrm{Y})$ & 2 to 5 & 5 to 15 \\
$\eta$ & $2.7810^{-3}$ & $2.7810^{-3}$ \\
\hline
\end{tabular}

At least four consecutive spectra are averaged prior to fitting. Since the fit for uncoalesced beams takes less than a fraction of a second on $\approx 2 \mathrm{GHz}$ Pentium IV processor, the system is able to report betatron tunes with a precision better than $1.10^{-4}$ and crude estimates of the linear chromaticity at a rate of $\approx 1 \mathrm{~Hz}$. The $\approx 1$ second-average tune spectra can be stored on a local disk for further offline analysis.

\section{RESULTS}

\section{Measurements of the chromaticity}

Such spectra are quite stable if the beam is circulating in the unperturbed Tevatron. Shown in Figure 1 are the tune spectra averaged over a period of two minutes from data taken in February 2005 with uncoalesced beam. The empirical Lorentzians fit the spectra remarkably well. The reason for this good agreement is not well understood. The value of the synchrotron frequency $\omega_{s}$ agrees well with the expected value.

From the spectra shown in Figure 1 we extract chromaticities $\left(Q_{x}^{\prime}, Q_{y}^{\prime}\right)=(7.6 \pm 1,2.7 \pm 0.3)$ in the horizontal and vertical planes respectively. The linear chromaticity was also measured shortly thereafter using the "classical" method, i.e by changing the rf frequency by $\approx 40$ $\mathrm{Hz}$, and recording the corresponding tunes. This method yielded the values $\left(Q_{x}^{\prime}, Q_{y}^{\prime}\right)=(8.6 \pm 0.1,3.5 \pm 0.05)$. The tunes were in good agreement with those recorded on the old HP3561 analyzers used routinely by the operators. The drawbacks with this classical method are that it is an invasive method since it moves the beam transversely and is often time consuming.

The precision of the method reported here is limited both by statistical and systematic errors. A simple analysis [10] shows that the number of spectra $N_{\text {spectra }}$ needed to obtain a relative error of $\Delta Q^{\prime} / Q^{\prime}$ follows $N_{\text {spectra }} \propto$ $1 /\left[\Delta Q^{\prime} / Q^{\prime}\right]^{2}$. For example, averaging over 30 spectra suffice to yield a relative error of $10 \%$ as long as the chromaticity $Q^{\prime}<6.5$. At injection in the Tevatron, chromaticities are in this range so fitted chromaticities can be obtained every 30 seconds. There are several systematic errors in this method as well, e.g. the chromaticity values extracted from the ratios $A_{1} / A_{0}$ and from $A_{2} / A_{0}$ from the first and second order synchrotron sidebands differ by about 0.5 (low $Q^{\prime}$ ) to 1 or 2 for $Q^{\prime} \approx 7$ to 10 . The spectra are also influenced by betatron coupling, nonlinear transverse and longitudinal motion - none of these effects are included in the expression in Equation 2. Another source of systematic error, yet un-accounted for, comes from the measurement of $\sigma_{t}$ : the Sampled Bunch Display (SBD) device has been optimized to report bunch lengths for coalesced beams which are two to three times longer. However, what matters for routine operations is that chromaticity measurements for a fixed beam yield reproducible values within a narrow range. The shape of these spectra is stable once the machine is tuned correctly and consequently the chromaticities followed over several minutes with this method typically occur within a spread of 1 unit.

Recently, a family of the octupole magnets provides additional Landau damping, allowing us to further reduce $Q^{\prime}$ and keep the beam stable[11]. This induces nonlinear effects, changes in the orbit and strong higher order chromaticities. For example the integral of $Q^{\prime \prime} d p$ over the momentum range corresponding to a $40 \mathrm{~Hz}$ rf frequency change is comparable to $Q^{\prime}$. When the octupoles are powered, the shape of the tune spectra changes. An example is shown in Fig. 2: the synchrotron lines broaden and the ratios $A_{ \pm k} / A_{0}, k \neq 0$ are no longer symmetric. Since the fit in Equation 2 predicts the synchrotron lines to have identical width and identical synchrotron tunes, the fit is not very good and the extracted $Q^{\prime}$ is difficult to interpret. We are currently including the higher order effects mentioned above in the analysis to improve the fit for such conditions. 

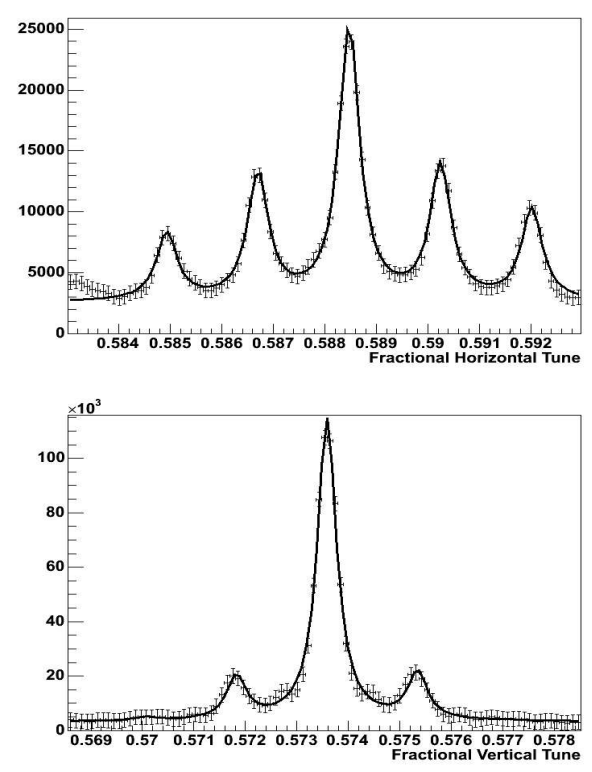

Figure 1: Horizontal (top) and Vertical (bottom) tune spectra of uncoalesced beams on the central orbit
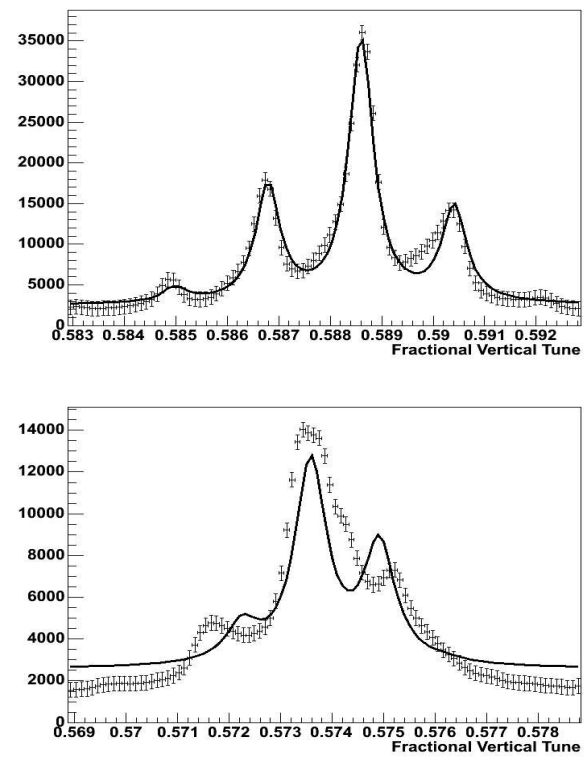

Figure 2: Horizontal (top) and Vertical (bottom) tune spectra obtained with uncoalesced beams on the central orbit. Octupole circuits are now on - other settings unchanged.

\section{Tune spectra for coalesced beams}

Tune spectra can also be recorded continuously during proton antiproton collider operation. (Fig. 3) Such spectra are quite complex due to (i) head-on beam-beam effects which increase the tune footprint but are different for proton and anti-protons (ii) long range beam-beam effects which affect different bunches in a beam differently [12]. Here, the relatively high $Q_{l}$ value of the $21.4 \mathrm{MHz}$ Schottky monitor is a disadvantage, as the device integrates over all bunches in a train. Also, the $21.4 \mathrm{MHz}$ resonator cavities, unlike the $1.7 \mathrm{GHz}$ system, have poor directionality, so we cannot distinguish between the two beam species. Yet, the evolution at high frequency is indicative of the antiproton tune drift.

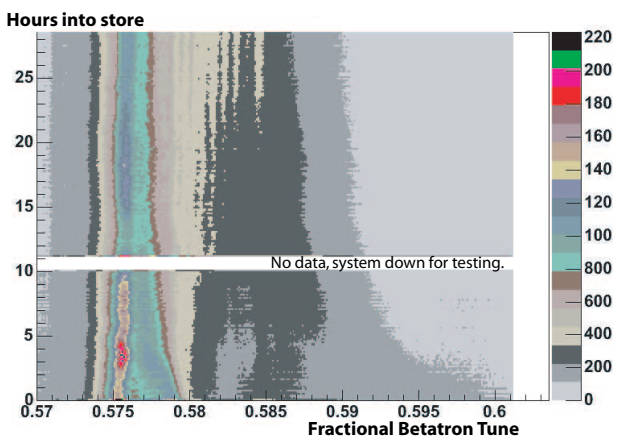

Figure 3: Water-fall tune spectra taken during a typical store.

In conclusion, improvements in electronics, Data Acquisition, offline software techniques and sufficient computing power allow us to run analysis of spectra routinely in real-time. Under optimal conditions, chromaticity measurements from the spectra of uncoalesced beams are in good agreement with values obtained by changing the rf frequency. This high quality data also allows a better determination of systematic uncertainties and potentially to characterize higher order effects.

\section{REFERENCES}

[1] C.Y. Tan, "Novel Tune Diagnostics for the Tevatron" PAC05, May 2005

[2] A. Jansson et al "Experience with the $1.7 \mathrm{GHZ}$ Schottky Pick-ups in the Tevatron", EPAC'04, July 2004, p. 2777

[3] G. Jackson "Tune Spectra in the Tevatron Collider", PAC 1989, Chicago, IL, p. 860

[4] V. Ranjbar and P.M. Ivanov "Measurement of Head-Tail Instability Parameters with a Fast Strip-Line Pickup in the Tevatron", PAC05, May 2005

[5] Interactive Circuit and Systems (Ltd), http://www.icsltd.com/

[6] The FNAL Accelerator Controls Mircroprocessor Group, http://www-ad.fnal.gov/controls/micro_p/micro_p.html

[7] M. Frigo and S.G. Johnson, http://www.fftw.org/

[8] R. Brun and F. Rademakers, Nucl. Inst. \& Meth. in Phys. Res. A 389 (1997) 81-86. See also http://root.cern.ch/.

[9] C.Y. Tan, X.L. Zhang and P. Lebrun, "Tune Measurement Methods of the Tevatron" PAC03, May 2003, p. 2703

[10] E. Todesco et al, FNAL Beams Document 1779 (2005)

[11] P.M. Ivanov et al, "Landau Damping of the Weak Head-Tail Instability at Tevatron” PAC05, May 2005

[12] T. Sen et al, Phys. Rev. ST Accel. Beams 7, 041001 (2004) 